This is the peer reviewed version of the following article:

Segatta, Francesco; Gdor, Itay; Rehault, Julien; Taioli, Simone; Friedman, Noga; Sheves, Mordechai; Rivalta, Ivan; Ruhman, Sanford; Cerullo, Giulio; Garavelli, Marco.

Ultrafast Carotenoid to Retinal Energy Transfer in Xanthorhodopsin Revealed by the Combination of Transient Absorption and Two-Dimensional Electronic Spectroscopy.

Chemistry. A European Journal, Volume 24, Issue 46, 26 July 2018, Pages 12084-12092.

which has been published in final form at https://doi.org/10.1002/chem.201803525

This article may be used for non-commercial purposes in accordance with Wiley Terms and Conditions for Use of Self-Archived Versions. 


\title{
Ultrafast Carotenoid to Retinal Energy Transfer in Xanthorhodopsin Revealed by the Combination of Transient Absorption and Two Dimensional Electronic Spectroscopy
}

\author{
Francesco Segatta, ${ }^{[a, b]}$ Itay Gdor, ${ }^{[c]}$ Julien Réhault, ${ }^{[\mathrm{d}]}$ Simone Taioli, ${ }^{[\mathrm{a}]}$ Noga Friedman, ${ }^{[\mathrm{e}]}$ Mordechai \\ Sheves, ${ }^{[\mathrm{e}]}$ Ivan Rivalta ${ }^{[\mathrm{f}]}$, Sanford Ruhman, ${ }^{*[\mathrm{c}]}$ Giulio Cerullo, ${ }^{[\mathrm{g}]}$ and Marco Garavelli ${ }^{*[\mathrm{~b}]}$
}

\begin{abstract}
By comparing two-dimensional electronic spectroscopy (2DES) and Pump-Probe (PP) measurements on xanthorhodopsin $(\mathrm{XR})$ and reduced-xanthorhodopsin (RXR) complexes, the ultrafast carotenoid-to-retinal energy transfer pathway is revealed, at very early times, by an excess of signal amplitude at the associated cross-peak and by the carotenoid bleaching reduction due to its ground state recovery. The combination of the measured $2 \mathrm{DES}$ and PP spectroscopic data with theoretical modelling allows a clear identification of the main experimental signals and a comprehensive interpretation of their origin and dynamics. The remarkable velocity of the energy transfer, despite the non-negligible energy separation between the two chromophores, and the analysis of the underlying transport mechanism, highlight the role played by the ground state carotenoid vibrations in assisting the process.
\end{abstract}

[a] Dr. F. Segatta, Dr. S. Taioli

European Center for Theoretical Studies in Nuclear Physics and Related Areas (ECT*-FBK)

38123 Trento, Italy

[b] Dr. F. Segatta, Prof. Dr. M. Garavelli

Department of Industrial Chemistry

University of Bologna

Viale Risorgimento 4, 40136, Bologna, Italy

E-mail: marco.garavelli@unibo.it

[c] Dr. I. Gdor, Prof. Dr. S. Ruhman

Department of Physical Chemistry

The Hebrew University

Jerusalem, 9190401, Istrael

E-mail: sandy@mail.huji.ac.il

[d] Dr. J. Réhault

Department für Chemie und Biochemie

University of Bern

Freiestrasse 3, 3012 Bern, Switzerland

[e] Dr. N. Friedman, Prof. Dr. M. Sheves

Department of Organic Chemistry

Weizmann Institute of Science

Rehovot 76100 , Israel

[f] Dr. I. Rivalta

Laboratoire de Chimie UMR 5182

Université Lyon, ENS de Lyon, CNRS, Unversité Lyon 1

Allée d'Italie 46, FR-69342 Lyon, France

[g] Prof. Dr. G. Cerullo

Department of Physics

Politecnico di Milano

Piazza Leonardo da Vinci 32, 20133, Milano, Italy

E-mail: giulio.cerullo@fisi.polimi.it

Supporting information for this article is given via a link at the end of the document.

\section{Introduction}

Xanthorhodopsin (XR) of the extremely halophilic eubacterium Salinibacter ruber [1] is a light-driven transmembrane proton pump with two chromophores: an all-trans-retinal (RET), responsible for the proton transport function, and a C40 carotenoid (CAR) salinixanthin [2,3], which acts as a lightharvesting antenna. It represents one of the simplest photosynthetic protein complexes for the study of excitation energy transfer (EET): a perfect donor-acceptor pair, with the carotenoid bound to the protein in a 1:1 ratio.

The complex excited state energy levels structure of the salinixanthin CAR includes the bright $S_{2}$ state, responsible for the strong absorption in the blue-green spectral region, and a dark state, $S_{1}$, lower in energy and populated via $S_{2}$-to- $S_{1}$ internal conversion (IC) on a sub-picosecond timescale. The existence of additional dark excited states (e.g., the so-called $S^{*}$ state), located below the $S_{2}$ state, has also been proposed in previous studies [4, 5]. In the protein-bound RET, the reactive $S_{1}$ state is known to undergo isomerization from all-trans to 13 -cis $[6,7]$.

The resolved XR crystal structure [8] reveals a $46^{\circ}$ angle between the two chromophores molecular axes, and a center to center distance of $11.7 \AA$. Steady-state spectroscopic studies $[9,10]$ were performed on both $\mathrm{XR}$ system and $\mathrm{NaBH}_{4}$-treated $\mathrm{XR}$, socalled reduced-xanthorhodopsin (RXR). The $\mathrm{NaBH}_{4}$ treatment blue-shifts the absorption energy of the RET, due to hydrogenation of the retinal Schiff base double bond, and prevents EET without significantly perturbing the CAR binding site. In both samples, well-resolved vibronic absorption bands of salinixanthin bound to $X R$ are recorded [11] (Figure 1). Action spectra for proton transport $[11,12]$ as well as fluorescence excitation spectra of the retinal chromophore [9] showed that light absorbed not only by the retinal but also by the carotenoid is employed for proton transport. Quantum yields of emission and energetic positioning of the chromophores states, indicate that the EET route should proceed from the extremely short-lived CAR $\mathrm{S}_{2}$ state to the reactive retinal $\mathrm{S}_{1}$. The efficiency of this process was estimated to be between $30 \%$ and $50 \%$ [9-12], suggesting an ultrafast EET process able to compete with the sub-picosecond CAR $S_{2} \rightarrow S_{1}$ IC

Femtosecond transient absorption studies [13-15] have been previously employed to directly investigate the dynamics of $\mathrm{CAR} \rightarrow \mathrm{RET}$ EET and CAR $\mathrm{S}_{2} \rightarrow \mathrm{S}_{1}$ IC. They provide a further support to the postulated EET pathway as well as an estimate of the various IC and EET rates. Polivka et. al [14], showed that the difference of the normalized XR and RXR transient absorption 
spectra at $1 \mathrm{ps}$ strictly resembles $\mathrm{J} / \mathrm{K}$ state signals of bacteriorhodopsin; moreover, they observed that a persistent signal at $615 \mathrm{~nm}$ was present at very long time $\left(t_{2}>20 \mathrm{ps}\right)$ in $X R$, while absent in RXR, explaining this difference as a signature of the RET K photoproduct. In the NIR, they also demonstrated that $\mathrm{S}_{2} \rightarrow \mathrm{S}_{2 \mathrm{n}}$ excited state absorption (ESA) signals last for about 100 fs in XR and $130 \mathrm{fs}$ in RXR (in line with the estimated $\sim 30 \%$ of EET efficiency), and characteristic RET stimulated emission (SE) was only present in the $\mathrm{XR}$ sample. Additional evidence was provided by the studies of some of the present authors $[13,15]$ : comparing PP experiments with different polarization it was possible to isolate sensitized RET signals in $\mathrm{XR}$, appearing around $560 \mathrm{~nm}$; the analysis of the $\mathrm{K}$ band anisotropy was also exploited to obtain an independent estimate of EET efficiency of $\sim 32 \%$. In the NIR, by comparing the relative intensities of $S_{1}$ evolution associated difference spectra (EADS), an estimate of EET efficiency around $35 \pm 7 \%$ was obtained.

These studies, performed with 100-fs [14] and 70-fs [15] time resolution, provided a CAR $S_{2}$ lifetime of $70-100 \mathrm{fs}$ and $110-130$ fs for XR and RXR respectively. Measurements performed in the visible window [13] with sub-10-fs time resolution estimated it to be below $30 \mathrm{fs}$, in contrast with the NIR [14,15] results, but it was not clear if broadband ultrafast excitation leads to fundamentally different $S_{2}$ excited states, or that nonlinearities dominate the extreme time resolution experiments [15].

By spreading the signals along two frequency axes, time resolved two-dimensional electronic spectroscopy (2DES) allows to both decongest transient absorption spectra and follow the direct signal of an EET process [16-18]. This appears in the 2DES maps as a cross-peak between the two interacting chromophores, whose form and evolution in time allows direct probe of its dynamics. For instance, this technique has been successfully applied to track and time resolve EET processes in complex multichromophoric LH systems $[16,19]$.

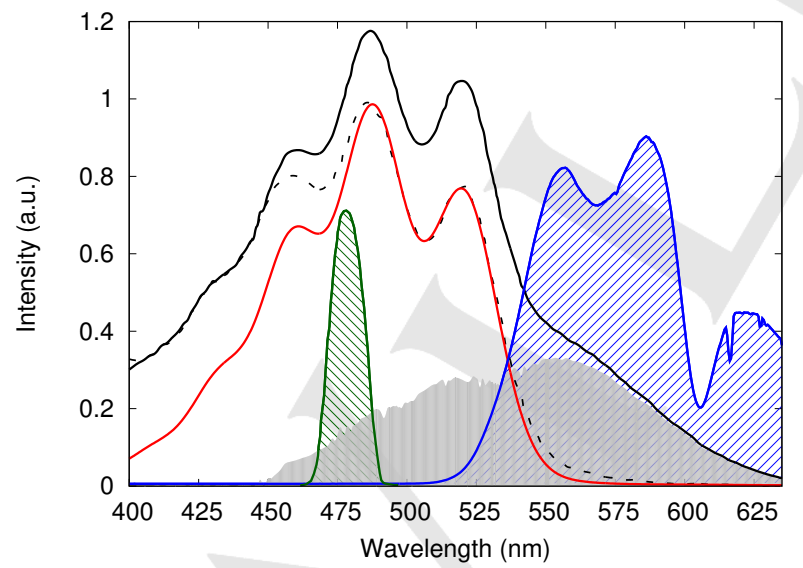

Figure 1. Experimental absorption spectra of XR (black solid line) and RXR (dashed line), together with their difference (grey filled curve), which reveals the RET absorption. The theoretical RXR absorption (red solid line) does not contain the reduced retinal contribution in the blue side of the spectrum. The spectra of the pulse used for the 2DES experiments (measured upstream of the sample, blue filled curve) and the pump pulse in the PP experiment (green filled curve) are also reported. The experimental absorption spectra were adapted from ref. 9 .
However, support by theoretical modeling is also mandatory to interpret the signals and disentangle the different components observed, specifically when spectrally congested regions persist [20].

In the present study, we compare experimental 2DES maps and broadband PP spectra of XR and RXR systems with simulated $R X R$ maps, aimed at finding signatures of the efficient $C A R \rightarrow R E T$ EET and elucidating the nature of the underlying transport mechanism. 2DES measurements (performed with a sub-10-fs time resolution and at room temperature) confirm that the EET route occurs from the bright $S_{2}$ state of the CAR to the $S_{1}$ of the $R E T$, clearly showing a EET related signature at the CAR-to-RET cross-peak. The comparison between 2DES and PP results, supported by theoretical arguments, allowed us to monitor the different processes that take place and follow their dynamics: in both samples, we observed the ultrafast $C A R S_{2} \rightarrow S_{1} / S^{*}$ internal conversion, revealed by the disappearance of the $S_{2} S E$ and by the rise of $S_{1} \rightarrow S_{1 n}\left(S^{*} \rightarrow S^{*}\right)$ ESA, together with the CAR $S_{2}$ spectral diffusion and the $S_{1}$ vibrational cooling; in $X R$, we tracked the carotenoid-to-retinal EET process, demonstrated by the excess of signal amplitude, at very early times, at the EET related cross peak, and by possible hot-GS photo-absorption signals, overlapping with the previously mentioned ESA. Our analysis enables to explore the role played by the CAR ground state vibrational levels in controlling and promoting the CAR $\rightarrow$ RET EET efficiency.

The Results and Discussion section is organized as follows: first we introduce the Spectral Overlap (between CAR emission and RET absorption) as a mean to describe the probability to populate the different CAR GS vibrational levels following the CAR $\rightarrow R E T$ energy transfer. Then we analyze the measured 2DES maps at few selected population times, enlightening additional evidences in favor of the ultrafast EET process. We discuss similarity and differences of 2DES and PP measurements, eventually summarizing the obtained results in a concise scheme (Figure 7).

\section{Results and Discussion}

The comparison between experimental absorption spectra of $X R$ and RXR and the theoretical CAR absorption curve is shown in Figure 1, together with the experimental intensity spectra of the pulses employed in 2DES and PP measurements: the former overlaps only with the tail of the CAR $S_{2}$ transition, while the latter is resonant with excited vibrational levels of the same transition. As previously reported $[9,14]$, the reduction of the retinal chromophore after $\mathrm{NaBH}_{4}$ treatment produces a quenching in the absorption for wavelength longer than $450 \mathrm{~nm}$, accompanied by an increase of the absorption below $450 \mathrm{~nm}$. The difference between the $X R$ and the RXR absorption (grey filled curve of Figure 1) therefore reveals, in this region of the spectrum, the retinal contribution to the overall $X R$ absorption. The theoretical $\mathrm{RXR}$ absorption spectrum matches the experimental one in the red side of the considered window, while it is considerable less intense in the blue side, where reduced retinal contributions (not accounted for in the model) are present. Note also that the negligible shift of the CAR transitions in XR and RXR indicates 


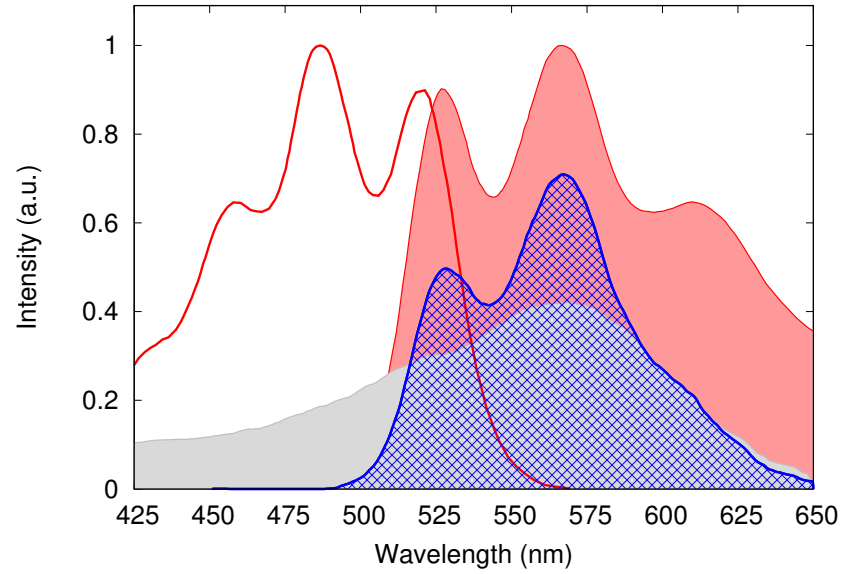

Figure 2. Experimental absorption spectra of RXR (red solid line) and its mirror image, representing the CAR $S_{2}$ fluorescence spectrum (red filled curve), together with RET absorption (grey filled curve). The Spectral Overlap, i.e. the integrand of Eq. (1), is depicted in blue. The experimental spectra were adapted from ref. 9

that CAR and RET are not forming a delocalized exciton state, as they maintain their individuality in both samples. This is further corroborated by the observed retention of carotene chirality in circular dichroism spectra [21,22] of both XR and RXR [15].

This observation points toward a Förster EET mechanism between localized CAR and RET states, which was previously postulated for the XR system [14,23]. Förster resonant energy transfer theory provides the EET rate when the interaction between the chromophores and the so called spectral overlap integral are known. The spectral overlap integral is given by

$$
J=\int F(v) A(v) / v^{4} d v
$$

where $F(v)$ and $A(v)$ are, respectively, the emission spectrum of the donor and the absorption spectrum of the acceptor (converted in energy scale and normalized to the unit area [24]). Figure 2 shows both the measured absorption of salinixanthin $S_{2}$ and its fluorescence, taken as the absorption mirror image [9], together with the computed spectral overlap.

The spectral overlap shown in Figure 2 (whose structure reflects the vibronic peaks of the CAR $S_{2}$ emission) allows for a qualitative estimate of the probability to populate the different possible CAR ground state (GS) vibrational levels following the CAR $\rightarrow R E T$ energy transfer. In particular, we note that a large percentage of the excited CAR molecules will not repopulate the original (cold) GS, but rather (hot) excited vibrational states of it. This remains true also when the measured salinixanthin $S_{2}$ fluorescence spectrum, which extends less in the red than the absorption mirror image, is employed for the spectral overlap calculation (see Figure $\mathrm{S} 1$ of the $\mathrm{SI}$ ). We remark here that it is not advisable to use the spectral overlap as a source of quantitative information. One should in fact consider that the CAR emission at very early times can differ from the CAR fluorescence profile, and can also be different when different pulse profiles are employed.

The analysis of the spectral overlap impacts the analysis of the experimental spectra in the following ways: i) the shape of the spectral overlap suggests that the maximum of the CAR-RET energy transfer related cross-peak in 2DES should appear around $520 \mathrm{~nm}$ pump and $560 \mathrm{~nm}$ probe. Moreover, as not all the excited CAR population involved in the energy transfer goes back to the vibrationally cold GS, one could observe ii) only a partial drop of the CAR GSB signal concurrent to energy transfer (i.e., not the expected $30-40 \%$ decay suggested by the EET efficiency, but just a fraction of it), and iii) the build-up of CAR hot GS photoinduced absorption signals in XR sample, which should appear as negative contributions at the red side of the $520 \mathrm{~nm}$ CAR GSB diagonal signal.
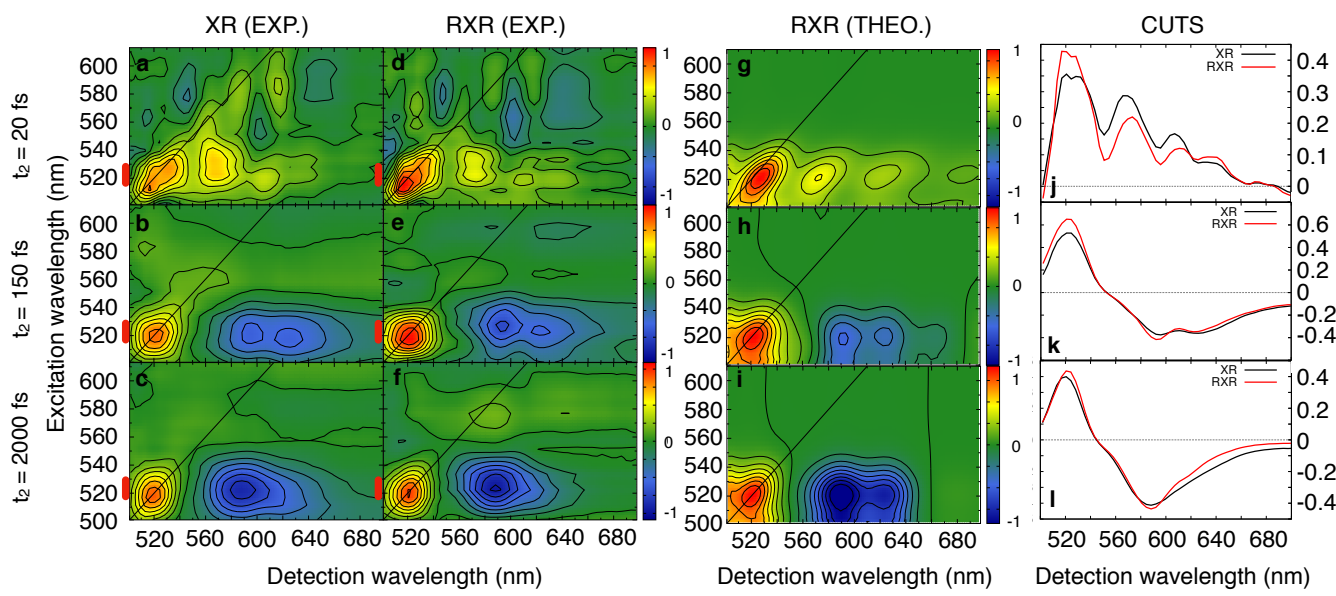

Figure 3. Experimental 2DES XR maps (a-c), experimental 2DES RXR maps (d-f), simulated RXR maps (g-i) and cuts of these maps (j-I) at different waiting times. The experimental maps were plotted between common XR and $R X R$ values ([-maximum,maximum]) at every selected $t_{2}$ time. $X R$ data were normalized so that, at $\mathrm{t}_{2} \sim 400 \mathrm{fs}$, the CAR GSB intensity (at the $520 \mathrm{~nm}$ diagonal) is the $85 \%$ of the RXR CAR GSB intensity. Theoretical maps were normalized in the range [maximum, maximum] (see Section S6 of the $\mathrm{SI}$ ) at every waiting time $\mathrm{t}_{2}$. The $2 \mathrm{DES}$ cuts were taken averaging the signal in the pump rage $515-530 \mathrm{~nm}$ (as highlighted by the red markers at the left side of the experimental maps). 
All of that considered, and to take into account differences in the samples optical densities and in the experimental conditions, we chose to renormalize XR signals in both 2DES and PP data, so that the $520 \mathrm{~nm}$ CAR signal after 400 fs (to be interpreted mainly as CAR S0 GSB, as CAR S2 SE should have already decayed at this time delay) is $85 \%$ of that of RXR (assuming an EET efficiency of $\sim 40 \%$ and that only $\sim 40 \%$ of the CAR molecules involved in this process goes back to the original GS, as suggested by the area below the first peak of the blue spectra in Figure 2). The results presented hereafter are shown to be robust against a range of possible $\mathrm{XR}$ scaling factors, as discussed in Section S2 of the SI.

Figure 3 presents experimental 2DES maps at three selected waiting times (t2 $=20,150,2000 \mathrm{fs}$ ) for the XR and the RXR samples (Figure $3 a-f)$, together with simulated RXR maps (Figure $3 \mathrm{~g}-\mathrm{i}$ ) and cuts (Figure $3 \mathrm{j}-\mathrm{I}$ ) of the 2DES maps obtained by averaging over a range of excitation wavelengths.

At $t_{2}=20 \mathrm{fs}$ (top row of Figure 3 ) the main detected signals in both samples are the positive GSB (diagonal peak at $520 \mathrm{~nm}$ ) and SE (sequence of peaks from the diagonal to the red side of the spectrum) of the salinixanthin $S_{2}$ state, which lasts for a few tens of femtoseconds. The comparison of XR and RXR cuts reveals a remarkable difference: an excess of signal amplitude in the $X R$ sample at the CAR-RET cross-peak (Figure $3 \mathrm{j}$ ), directly revealing, as discussed afterwards, the presence of the underlying EET process. At $\mathrm{t}_{2}$ times around $100-200 \mathrm{fs}$ (central row in Figure 3 ) we observe the positive salinixanthin $\mathrm{S}_{2}$ GSB band, which becomes more symmetric, from the initially elongated form, due to spectral diffusion, and the well-known negative salinixanthin $S_{1}$ (the elongated blue peak in the red side of Figure $3 \mathrm{~b}, 3 \mathrm{e}$ and $3 \mathrm{~h}$ ) and $S^{*}$ (the blue peak around $585 \mathrm{~nm}$ probe in the same panels of Figure 3) ESA signals, in the interval $550-750 \mathrm{~nm}$, which dominate and cover the carotenoid-retinal cross-peak and the $\mathrm{CAR}_{2} \mathrm{SE}$ region. The comparison of the cuts (Figure $3 \mathrm{k}$ ) shows an unexpected similarity of the ESA signals intensity in the two samples, in contrast with the notion that the RXR CAR $S_{1}$ (and $S^{*}$ ) should have been populated more than in $X R$, being $I C$ the unique deactivation channel for CAR $S_{2}$ in the former system. Following the analysis of the spectral overlap, the observation that a large percentage of the CAR molecules involved in the EET process will populate excited vibrational levels of the CAR GS, rather than the original cold GS, suggests a possible explanation of this similarity: photoinduced absorption from this vibrationally hot GS will indeed result in a negative signal that appears red-shifted with respect to the salinixanthin GS absorption (Figure 4c). This signal superimposes constructively with the negative $S_{1} / S^{*} E S A$, and might explain the enhanced intensity of $X R$ features in that spectral region. Furthermore, the ultrafast nature of the carotenoid-to-retinal EET process is also understood in terms of the improved energy resonance condition between the two molecules [25] which is guaranteed when the vibrational excited states of CAR GS are populated: this is clearly suggested by looking at the spectral overlap profile of Figure 2, which shows that the largest overlap between RET $S_{1}$ absorption and CAR $S_{2}$ emission involves the second vibronic band of the latter.

At longer times $\left(t_{2}>1 \mathrm{ps}\right.$, bottom row in Figure 3 ) we still observe the carotenoid $S_{0}$ GSB signal on the diagonal and the $S_{1}\left(S^{*}\right)$ ESA cross peak, which has been narrowed and blue-shifted due to $S_{1}$ vibrational cooling. In the XR complex, at this delay time, we also expect to see the photoinduced absorption signals of the $\mathrm{J}$ (and early populated K) photo-product state of RET as previously suggested by Polivka et al. [14], which is indeed revealed by the clear increase of signal in the XR cut.

In Figure 4, we present a comparison between experimental and theoretical differences of the XR and RXR cuts (obtained by averaging the signals in the $515-530 \mathrm{~nm}$ pump wavelength window) at early times ( $\left.t_{2}=20 \mathrm{fs}\right)$. The theoretical $X R$ curve only considers CAR contributions, accounting for the shorter lifetime of the $S_{2}$ state due to the presence of the EET channel. We show that the prediction of a model which excludes both direct and indirect retinal contributions at pump wavelength corresponding to salinixanthin $S_{2}$ excitation (red dashed curve in Figure 4c), is qualitatively different with respect to experimental measurements (black solid curve in Figure 4c). On the contrary, the addition of a retinal-like GSB/SE contribution (modeled via a gaussian function centered around the retinal absorption maximum, the grey filled curve of Figure 4c), turns out to reproduce the main features of the XR/RXR difference. The introduced gaussian contribution represents a signal of the right sign (positive) and at the right position (EET related CAR-RET cross-peak) to qualitatively resemble a sensitized retinal GSB/SE signal. We assign to the EET related signal the excess of signal amplitude observed for the $520 \mathrm{~nm} / 560 \mathrm{~nm}$ cross-peak in the XR sample. To support this assignment, we discuss in detail the origin of all the signals which could in principle contribute to this spectral region at early times, when pumping the carotene chromophore (between $510-530 \mathrm{~nm}$ ) and probing in the 500-750 $\mathrm{nm}$ window.

The presence of possible carotene $S_{2} \rightarrow S_{2 n}$ ESA signals cannot be excluded, but to the best of our knowledge it has never been reported in studies of similar carotenoid systems in the visible

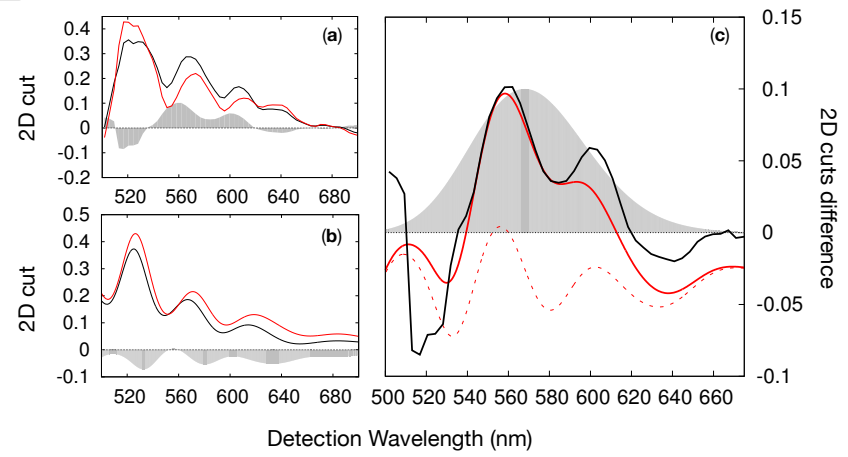

Figure 4. Differences of $X R$ and RXR 2DES cuts obtained by averaging excitations wavelengths in the $515-530 \mathrm{~nm}$ interval at $t_{2}=20 \mathrm{fs}$. Experimental (a) and theoretical (b) XR and RXR cuts (red and black solid line respectively) together with their differences (grey filled curves). Since in the theoretical maps RET contributions are not considered, the differences between the two curves are only given by the different $S_{2}$ states lifetimes (which control the $S_{2} S E$ disappearance, and produce the lifetime broadening of the transitions) and by the imposed XR CAR GSB scaling described in the main text (XR CAR GSB at $400 \mathrm{fs}$ is the $85 \%$ of the RXR CAR GSB). Panel (c): experimental difference (black solid line) and theoretical difference, before (red dashed line) and after (red solid line) the sum with the retinal GSB/SE component (grey filled curve). 
region (they are instead observed in the near-infrared region, around $1200 \mathrm{~nm}$ [15]). We exclude CAR $S_{1} \rightarrow S_{1 n}$ (and corresponding $S^{*} \rightarrow S^{*}$ ) ESA signals, which are known to appear only at later times. Contributions from direct retinal excitation (which has a non-negligible absorption at $520 \mathrm{~nm}$ ) are also of minor importance: direct retinal contributions are barely detected, in 2DES maps, at the maximum of the retinal absorption (a very weak signal is measured in the diagonal around $560 \mathrm{~nm}$, as shown in Figure S3 of the SI), being therefore even weaker for wavelengths around $520 \mathrm{~nm}$ at which the retinal absorbs less. At variance, indirect RET signals (i.e. signals coming from RET populated via EET from the CAR S2) are much more intense, as they are weighted with the CAR S2 dipole strength (see Section $\mathrm{S} 4, \mathrm{SI})$. To this respect, we note that the ability of monitoring both direct and indirect (sensitized) RET signals in different spectral regions of the same recorded maps is a considerable advantage of 2DES over PP experiments.

One could expect to observe, due to the presence of a finite carotenoid-retinal coupling, cross-peaks in the CAR-RET off diagonal region, both above and below diagonal. We do not observe any signal in the above diagonal region, and we can exclude possible contributions also in the region of interest: due to the negligible delocalization of the excitation on the two chromophores we expect a cancelation of coupling related offdiagonal signals. It is worth noting that a fast disappearance of the XR-RXR differential signal at this $(520-560 \mathrm{~nm})$ cross-peak is also observed. This can be easily accounted for considering the underlying fast evolution of the retinal chromophore when promoted to its $S_{1}$ state by the EET process: in fact, if at short times the retinal SE (appearing during the carotene-to-retinal EET process) should be similar to the retinal GS absorption, it very quickly undergoes a pronounced red-shift, disappearing from the probed window, as previously observed for other rhodopsin systems in correspondence to isomerization $[26,27]$. Moreover, negative contributions from the salinixanthin $S_{1}\left(S^{*}\right) E S A$ will progressively gain intensity at increasing $t_{2}$ times, thus masking the cross peak (the evolution of the involved states is also reported in Figure 7d). All that in mind, it is apparent that the above described signals prove indirect excitation of the retinal chromophore via ultrafast EET from the carotene moiety that has been directly excited by light.

In Figure 5 we compare PP signals ("PP - 480" and "PP - 520", with pump pulses centered at 480 and $520 \mathrm{~nm}$, respectively) and 2DES cuts (obtained by averaging the signals for excitation wavelengths between 500 and $550 \mathrm{~nm}$ ) for both XR and RXR at early times. For each experiment, $\mathrm{XR}$ data were normalized as previously explained.

The 6 graphs look very similar, as they are all dominated by CAR related signals. In all the three experiments CAR $S_{2}$ GSB and $\mathrm{S}_{1} / \mathrm{S}^{*} \mathrm{ESA}$ signals are recorded, together with CAR $\mathrm{S}_{2} \mathrm{SE}$, visible at $30 \mathrm{fs}$ as a sequence of positive peaks in the 530-650 nm interval; one can also observe in all the samples an excess of signal intensity in the XR sample around $560 \mathrm{~nm}$ at $30 \mathrm{fs}$, here identified (also thanks to the ability of 2DES of resolving the signals over an additional -excitation- energy axis) as a signature of the CAR $S_{2}$ to RET $S_{1}$ EET.

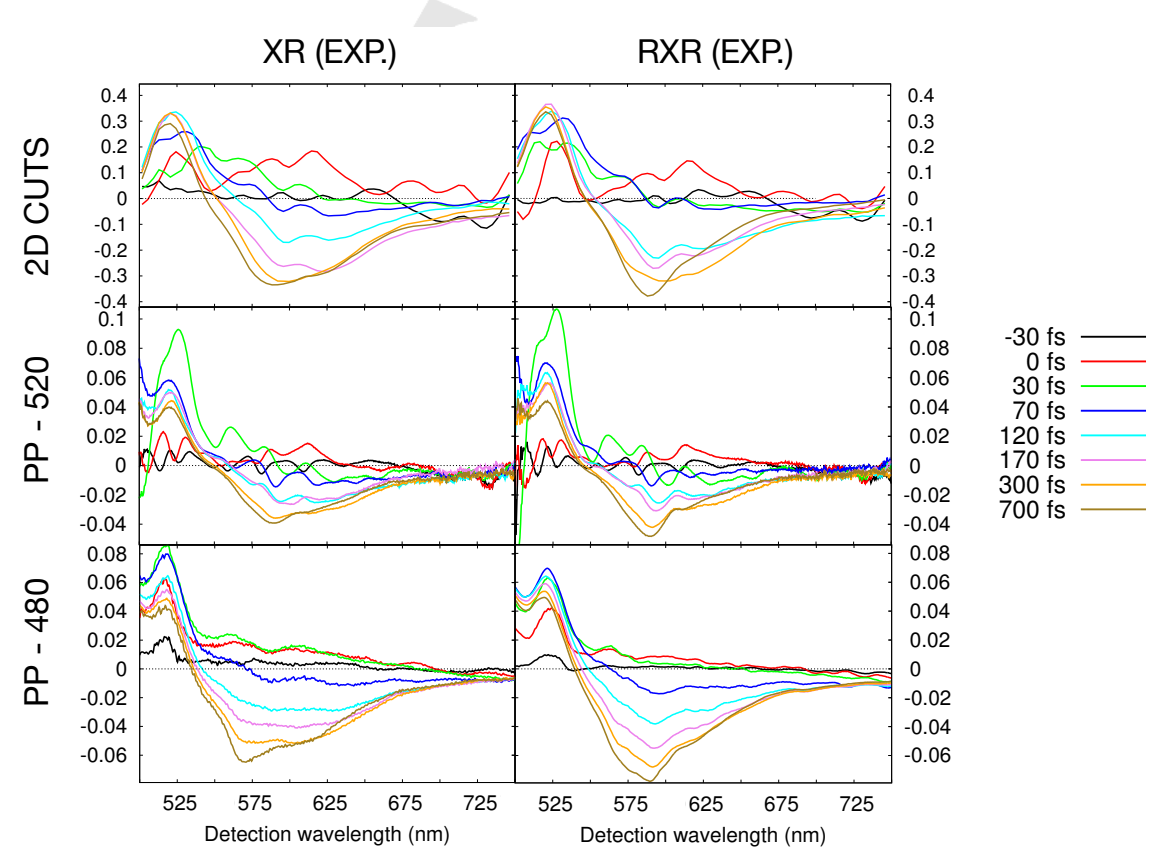

Figure 5. Comparison of XR (left column) and RXR (right column) experimental 2DES cuts and PP spectra at selected time delays. All the curves were normalized so that the XR CAR GSB at 400 fs is the $85 \%$ of the RXR CAR GSB, as described in the text. The data were plotted with a sign convention consistent with the one of the 2DES maps: GSB and SE signals are positive, while ESA signals are negative. 

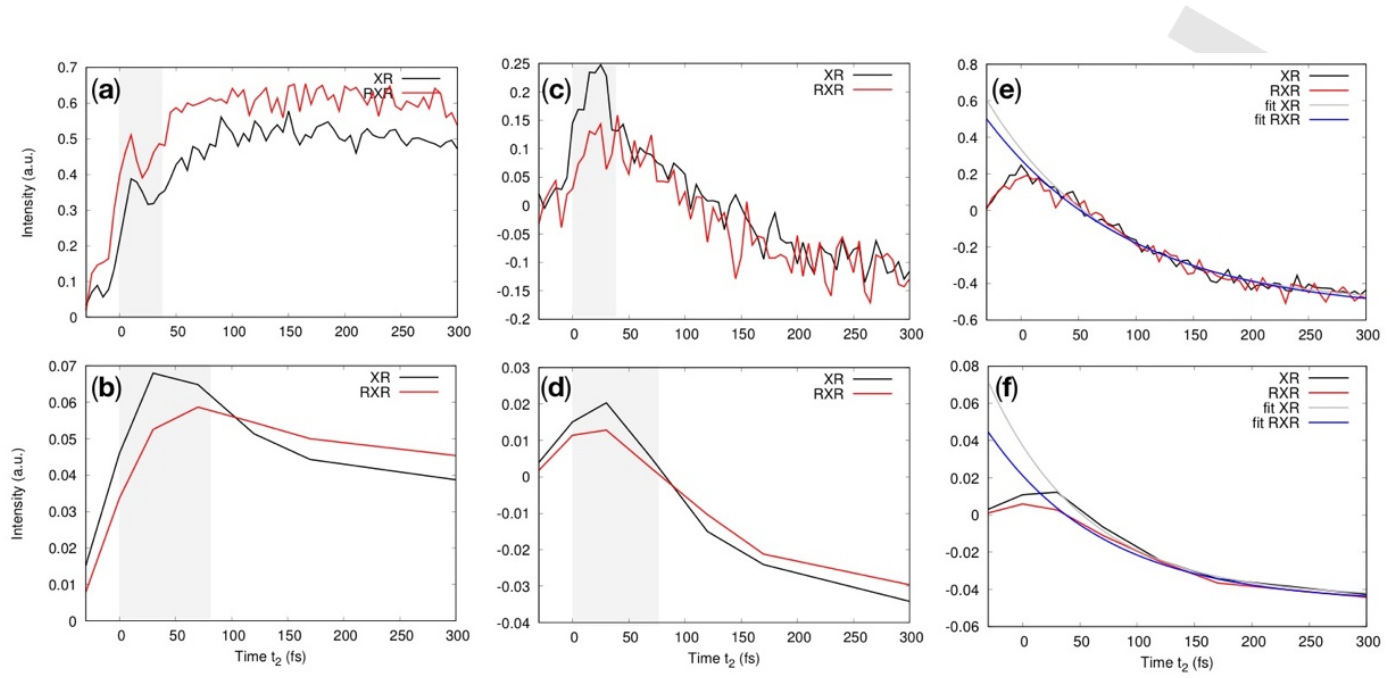

Figure 6. Evolution of selected regions of 2DES maps (first row) and PP data (second row) along the population time $\mathrm{t}_{2}$. (a) $2 \mathrm{DES}$ region within $520-530 \mathrm{~nm}$ pump and 520-530 probe (CAR GSB and SE); (b) PP evolution at $520 \mathrm{~nm}$; (c) 2DES region within 520-530 nm pump and 560 probe (CAR SE and EET cross-peak); (d) $\mathrm{PP}$ evolution at $560 \mathrm{~nm}$; (e) 2DES region within $520-530 \mathrm{~nm}$ pump and 610 probe $\left(\mathrm{S}_{1} \rightarrow \mathrm{S}_{1 \mathrm{n}} \mathrm{ESA}\right)$; the fits give a build-up of the ESA peak with time constants of 108 fs and $127 \mathrm{fs}$ for XR and RXR, respectively; (f) PP evolution at $615 \mathrm{~nm}$; the fits give a build-up of the ESA peak with time constants of $85 \mathrm{fs}$ and $102 \mathrm{fs}$ for XR and $\mathrm{RXR}$, respectively. The shadows highlight region of interest in the various plots. All the curves were normalized so that the XR CAR bleaching at $400 \mathrm{fs}$ is the $85 \%$ of the RXR CAR bleaching, as described in the text.

Differences in the various experiments in the $\mathrm{max} / \mathrm{min}$ ratio at each given time delay can be attributed to the different pulse polarization setups. We also note that the positioning of some peaks (e.g., CAR GSB and max of S1/S* ESA) is nearly the same for $2 D$ cuts and $P P-520$, while these are slightly blue-shifted in the PP -480 XR sample. Finally, one of the most remarkable differences is related to the dynamics of the $520 \mathrm{~nm}$ peak (Figure $6(a-b))$ in both PP data the signal increases, reaches a maximum (where CAR S2 GSB and SE constructively add together) and then quickly decays (due to the CAR SE decay), while in the 2DES the peak experiences a different dynamics, as if a negative signal was temporarily overlapping in that region. A similar dynamics of the peak was observed in ref. 18 , for $\beta$-carotene, where a comparable pulse setup, overlapping with the red tail of the $\beta$-carotene absorption spectrum, was employed. There is no simple explanation why PP and 2DES signals obtained employing the same pulse shapes and polarizations should display different peaks dynamics. It has recently been demonstrated that the effect of the laser spectral profile on the observed spectral features in 2DES is highly non-trivial, and attention should be paid to avoid signal misinterpretation [28]. To this respect, the peculiar dynamics of the CAR $520 \mathrm{~nm}$ peak has to be further investigated. In Figure 6 we also report the evolution of the signal intensity in the $520 \mathrm{~nm}-560 \mathrm{~nm}$ region (EET related cross-peak, Figure 6(cd)), highlighting the excess of $X R$ signal amplitude with a grey shadow, and in the $520 \mathrm{~nm}-610 \mathrm{~nm}$ region $\left(S_{1} \rightarrow S_{1 \mathrm{n}}\right.$ signals, Figure 6(e-f)); the fits show that in both 2DES and PP data the build-up of this ESA signal is faster in XR than in RXR, as expected from the fact that the lifetime of the CAR $S_{2}$ state is shorter in the native system than in the reduced one. This represents another indirect proof of the occurrence of EET in XR. A comprehensive scheme of the states and the signals involved in a 2DES experiment on XR, is depicted in Figure 7, and it summarizes some of the obtained results: Figures 7 (b) shows that the resonant donor-acceptor condition is enhanced by vibrationally excited levels of the GS of the donor (salinixanthin). Figure 7(c) shows both the red-shift of the RET emission over time, the $S_{1} \rightarrow S_{1 n}\left(S^{*} \rightarrow S^{*}\right)$ ESA following IC, and the hot CAR GS absorption following EET. Figure 7(e) summarizes the evolution of states population in both $\mathrm{XR}$ and $\mathrm{RXR}$, as obtained within a simple rate equation model. The model includes the ultra-short signal lifetime of the "Franck-Condon" $S_{1}$ state of the retinal, set to $30 \mathrm{fs}$ (following the arguments of ref. 26). The fast evolution of the RET on its potential energy surface, together with the emerging CAR $S_{1}$ (and $S^{*}$ ) ESA signals, explains the fast disappearance of EET related RET signals around $560 \mathrm{~nm}$ in the 2DES maps.

\section{Conclusions}

In conclusion, we have compared 2DES and PP measurements performed on $\mathrm{XR}$ and $\mathrm{RXR}$ systems. The identification and interpretation of the recorded signals in both spectroscopic techniques, supported by a theoretical analysis, allowed us to provide additional evidence of the ultrafast carotenoid-to-retinal EET process at very early population times $t_{2}$. Interestingly, our analysis uncovers the importance of vibrationally excited levels of 

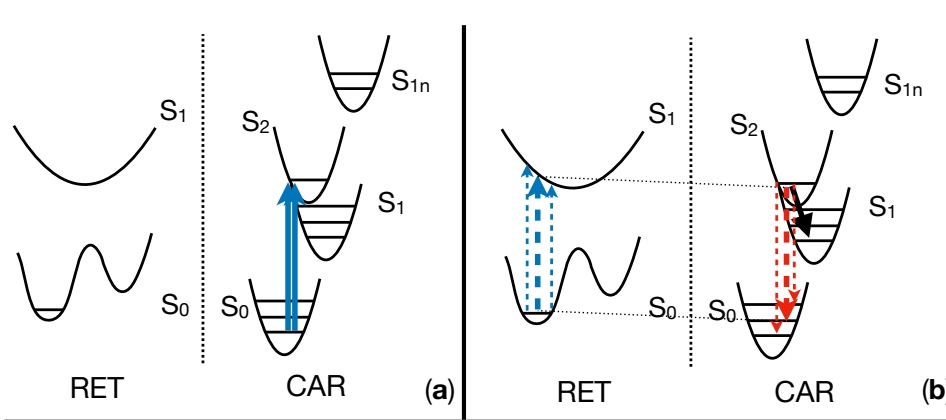

and evolution along $t_{3}$

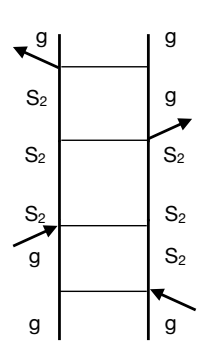

SE CAR $\mathrm{S}_{2}$

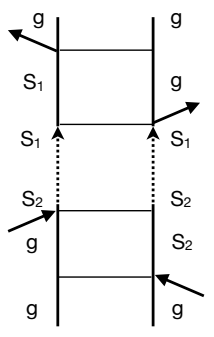

SE RET $\mathrm{S}_{1}$

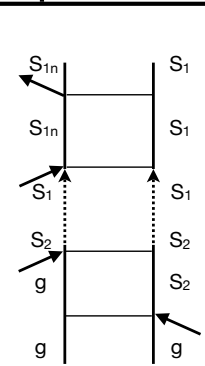

ESA CAR S (d) XR CAR-S2 XR CAR-(S1\&S*)
XR RET-S1(FC)

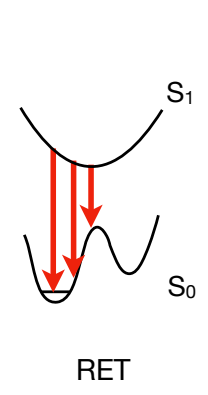

So

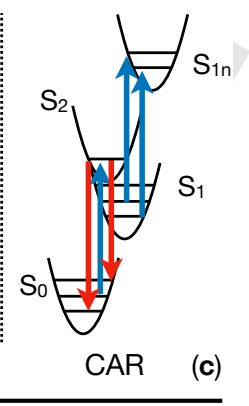

RXR CAR-S2 - - RXR CAR-(S1\&S*) - -

Figure 7. (a-c) Energy level diagrams depicting the main transitions occurring during times $t_{1}, t_{2}$ and $t_{3}$ : (a) excitation of the salinixanthin $S_{2}$ state (the direct retinal excitation, which is a minor component, is not shown), (b) IC (black solid arrow) and EET (upwards and downwards dashed arrows) processes activated after excitation of salinixanthin in XR (the energy matching condition for EET is maximized when hot CAR GS vibrations are populated, as highlighted by the different arrow thickness), (c) main SE (both in CAR $S_{2}$ and in RET $S_{1}$ ), and ESA signals $\left(S_{1} \rightarrow S_{1 n}\right)$; blue arrows denote absorption, while red arrows emission; only the $S_{1}$ state is shown for clarity (similar schemes hold for CAR $S^{*}$ ), and for the same reason only one effective mode is depicted in the PES; (d) (Rephasing) Feynman diagrams of the possible processes activated after population of CAR $S_{2}$ state (a population preserving diagram, giving rise to $S_{2}$ SE, and two population changing diagrams, giving rise to EET related SE and IC related ESA); the dashed arrows indicate that population is changing during $t_{2}$. Similar considerations hold for nonrephasing diagrams. (e) Population dynamics of the spectroscopic relevant states in XR (solid lines) and RXR (dashed lines), for a model in which EET has $\sim 40 \%$ efficiency.

the carotenoid electronic ground state in assisting the ultrafast $\mathrm{CAR} \rightarrow \mathrm{RET}$ EET process, as they provide improved energy matching and increased transition probability. This model is supported by the analysis of the CAR $S_{2}-R E T S_{1}$ spectral overlap, which suggests that during the energy transfer process the CAR $\mathrm{S}_{0}-\mathrm{S}_{2}$ GSB signal will not drop by the expected $30-40 \%$ (i.e., the efficiency of the EET process), but only by a fraction of it, eventually allowing to explain the presence of additional negative signals at the red side of the CAR $S_{2}$ bleaching as the absorption from a hot GS CAR to $S_{2}$. The impact of the different excitation pulse spectra on this analysis and on the interpretation of the recorded signals has to be further investigated.

Finally, we note that despite the simplicity of the system (a donoracceptor couple), the obtained maps are highly congested by overlapping spectral features, and that theoretical modeling is needed for signals identification.

\section{Experimental Section}

Sample Preparation: XR protein was extracted from S. Ruber (the growth was carried out according to previously published methods [1]). XR membranes samples were isolated using previously described procedures [29]. The XR and RXR samples were prepared in $0.1 \mathrm{M} \mathrm{NaCl}$ and $50 \mathrm{mM}$ Tris buffer, at $\mathrm{pH}=8.4$.

Reduction of the protonated Schiff-base bond with $\mathrm{NaBH}_{4}$ : To a solution of native XR (50 mM Tris buffer, $\mathrm{pH}=8.4,300 \mathrm{mM} \mathrm{NaCl}, 30 \%$ sucrose), $0.12 \mathrm{M}$ of $\mathrm{NaBH}_{4}$ were added. The reduction process was carried out using illumination for $1 \mathrm{~h}$. The light was filtered through a long pass cutoff filter with $\lambda \max >550 \mathrm{~nm}$ (Schott, Mainz, Germany) to prevent over heating of the sample. The sample was dialyzed against $0.1 \mathrm{M} \mathrm{NaCl}$, to remove $\mathrm{NaBH}_{4}$

PP setup: A room temperature sample was syringe pumped through a 0.4 $\mathrm{mm}$ path length flow cell equipped with $0.1 \mathrm{~mm}$ glass windows. The concentrations of XR and RXR in buffer produced nominal OD of 0.7 at $480 \mathrm{~nm}$. The integrity of the sample was determined by measuring its absorption spectrum before and after each run. 30 fs pulses at $790 \mathrm{~nm}$ 
were derived from a homemade multipass amplified Ti:Sapphire laser system. A few $\mu \mathrm{J}$ were used to generate a multi-filament white light continuum probe by focusing in $3 \mathrm{~mm}$ of sapphire. The continuum pulses were collimated and re-focused into the sample with reflective optics. The remaining fundamental was used to pump an optical parametric amplifier (OPA, TOPAS, Light Conversion) and produce $30 \mathrm{fs}$ pump pulses centered at $480 \mathrm{~nm}$ by mixing signal with OPA fundamental. The pump chirp was compensated in a slightly misaligned zero dispersion grating pulse shaper $250 \mathrm{~nJ}$ of the resulting pulse was focused in the sample to a spot of $\sim 200$ um in diameter. Kerr scans in water provided the probes' wavelength dependent group delay, and indicated a pump-probe cross-correlation of $\sim 70$ fs throughout the probed range. After the sample, probe and reference pulses were collected by fibers into a double spectrometer setup to generate the time-dependent differential transmission spectra. The resulting spectra were corrected for group delay dispersion of the probe continuum.

2DES setup: The principle of operation of our 2DES setup is described extensively elsewhere $[30,31]$. 2DES in the partially linear pump-probe geometry can be seen as an extension of conventional PP spectroscopy, where two identical collinear pump pulses are used and their delay $t_{1}$ (coherence time) is scanned in time, for a fixed value of the probe pulse delay $t_{2}$ (population time). The probe pulse is dispersed in a spectrometer, providing resolution in the detection frequency. The Fourier transform with respect to the pump pulses delay provides the resolution of the signals with respect to the excitation frequency. The 2DES setup starts with a noncollinear OPA (NOPA) generating sub-10-fs visible pulses with spectrum extending from 500 to $720 \mathrm{~nm}$. The NOPA output is divided by a beam splitter ( $90 \%$ transmission, $10 \%$ reflection) into pump and probe lines. The identical and phase-locked pair of femtosecond pump pulses is generated by the Translating-Wedge-Based Identical-Pulses-eNcoding System (TWINS) technology $[30,31]$. TWINS uses birefringence to impose usercontrolled temporal delays, with attosecond precision, between two orthogonal components of broad-bandwidth laser pulses. Rapid scanning of the inter-pulse delay allows robust and reliable generation of 2DES spectra. In order to determine zero delay between the pump pulses and properly phase the 2DES spectra, part of the pump beam is split off and sent to a photodiode to monitor the interferogram of the pump pulse pair The additional dispersion introduced by the TWINS on the pump pulse pair is compensated by a suitable number of bounces on a pair of chirped mirrors. Pump and probe pulses are non-collinearly focused on the sample and the transient transmission change is measured on a CCD camera in a spectrometer. The polarization between pump and probe pulses was set to perpendicular with thin polarizers to minimize pump scattering signals on the CCD.

\section{Theoretical Section}

The electronic structure parameters of the $S_{2}$ state of the salinixanthin (CAR) moiety in both XR and RXR, were tuned to fit the red side of the experimental RXR absorption spectrum (less affected by the presence of the reduced retinal absorption). The main ingredients of the model include vertical excitation energy, transition dipole moment, and the strong intramolecular $C-C$ and $C=C$ stretching modes (centred at $1155 \mathrm{~cm}^{-1}$ and 1520 $\mathrm{cm}^{-1}$, respectively, in accordance with the Fourier analysis of XR spectral modulations [13]), responsible for the pronounced vibronic structure observed in the linear spectra (Figure 1). The lifetime of the Car $S_{2}$ state was accounted for, and set to 120 fs in the RXR (matching the $S_{2}$-to- $S_{1}$ internal conversion rate experimentally observed [14]) and to $70 \mathrm{fs}$ in the XR (assuming a $40 \%$ efficiency for the carotenoid-to-retinal EET rate). Higher energy carotenoid excited states were also included in the model to reproduce the measured ESA signals $\left(S_{1} \rightarrow S_{1 n}\right.$ and $S^{*} \rightarrow S^{*}$ transitions), and their parameters were fine-tuned to reproduce $2 \mathrm{DES}$ cuts at early times. All the model parameters are summarized in the SI (Tables S1, S2 and S3). The coupling of all the transitions to the continuum bath was also modeled through a spectral density (adapted from ref. 18), and the static disorder of the $S_{2}$ excitation energy was set to $260 \mathrm{~cm}^{-1}$. In the simulation of 2DES maps, we have accounted for the finite time duration of the pulses by convoluting the maps (along $t_{2}$ ) with a gaussian function, whose width ( $\sigma=8 \mathrm{fs}$ ) was determined as the time-width of the pulses. The pulse polarization was set to $\mathrm{VVHH}$ as in the experiment. A Stokes shift of 100 $\mathrm{cm}^{-1}$ for the RXR-CAR and $50 \mathrm{~cm}^{-1}$ for the XR-CAR, was applied to the simulated Stimulated Emission signals, to reproduce the 2DES cuts at early times.

\section{Acknowledgements}

This work was supported by the European Research Council Advanced Grant STRATUS (ERC-2011-AdG No. 291198) and the H2020 Grant Agreement number 765266 (LightDyNAmics).

Keywords: Two-Dimensional Electronic Spectroscopy • Förster Energy Transfer $\bullet$ ground state vibrations $\bullet$ Retinal Proteins $•$ Carotenoids

[1] J. Antón, A. Oren, S. Benlloch, F. Rodríguez-Valera, R. Amann, R. Rosselló-Mora, International Journal of Systematic and Evolutionary Microbiology 2002, 52(2), 485-491

[2] B. F. Lutnaes, A. Oren, S. Liaaen-Jensen, Journal of Natural Products 2002, 65(9), 1340-1343

[3] T. Polívka, V Sundström, Chemical Reviews 2004, 104(4), 2021-2072

[4] Y. Koyama, F. S. Rondonuwu, R. Fujii, Y. Watanabe, Biopolymers 2004, 74(1-2), 2-18

[5] C. C. Gradinaru, J. T. M. Kennis, E. Papagiannakis, I. H. M. van Stokkum, R. J. Cogdell, G. R. Fleming, R. A. Niederman, R. van Grondelle, Proceedings of the National Academy of Sciences 2001, 98(5), 23642369

[6] R. A. Mathies, S. W. Lin, J. B. Ames, W. T. Polland, Annual Review of Biophysics and Biophysical Chemistry 1991, 20(1), 491-518

[7] R. Mathies, C. Brito Cruz, W. Pollard, C. Shank, Science 1988, 240(4853), 777-779

[8] H. Luecke, B. Schobert, J. Stagno, E. S. Imasheva, J. M. Wang, S. P. Balashov, J. K. Lanyi, Proceedings of the National Academy of Sciences 2008, 105(43), 16561-16565.

[9] S. P. Balashov, E. S. Imasheva, J. M. Wang, J. K. Lanyi, Biophysical Journal 2008, 95(5), $2402-2414$

[10] J. K. Lanyi, S. P. Balashov, Biochimica et Biophysica Acta (BBA) 2008 , 1777 (7-8), 684-688

[11] S. P. Balashov, E. S. Imasheva, V. A. Boichenko, J. Antón, J. M. Wang, J. K. Lanyi, Science 2005, 309(5743), 2061-2064.

[12] V. A. Boichenko, J. M. Wang, J. Antón, J. K. Lanyi, S. P. Balashov, Biochimica et Biophysica Acta (BBA) - Bioenergetics 2006, 1757(12), $1649-1656$

[13] J. Zhu, I. Gdor, E. Smolensky, N. Friedman, M. Sheves, S. Ruhman, The Journal of Physical Chemistry B 2010, 114(8), 3038-3045

[14] T. Polívka, S. P. Balashov, P. Chábera, E. S. Imasheva, A. Yartsev, V. Sundstr, Biophysical Journal 2009, 96(6), 2268 - 2277.

[15] I. Gdor, I. Zhu, B. Loevsky, E. Smolensky, N. Friedman, M. Sheves and S. Ruhman, Physical Chemistry Chemical Physics 2011, 13, 3782-3787

[16] T. Brixner, J. Stenger, H. M. Vaswani, M. Cho, R. E. Blankenship, G. R. Fleming, Nature 2005, 434(7033), 625-628

[17] G. S. Schlau-Cohen, A. Ishizaki, G. R. Fleming, Chemical Physics 2011, 386(1-3), 1-22 
[18] N. Christensson, F. Milota, A. Nemeth, J. Sperling, H. F. Kauffmannm T. Pullerits, J. Hauer, The Journal of Physical Chemistry B 2009, 113(51) 16409-16419

[19] E. Collini, C. Y. Wong, K. E. Wilk, P. M. G. Curmi, P. Brumer, G. D. Scholes, Nature 2010, 463, 644-647

[20] F. Segatta, L. Cupellini, S. Jurinovich, S. Mukamel, M. Dapor, S. Taioli, M. Garavelli, B. Mennucci, Journal of the American Chemical Society 2017, 139(22), 7558-7567

[21] E. S. Imasheva, S. P. Balashov, J. M. Wang, J. K. Lanyi, The Journal of Membrane Biology 2010, 239(1-2), 95-104

[22] E. S. Koganov, V. Brumfeld, N. Friedman, M. Sheves, The Journal of Physical Chemistry B 2014, 119(2), 456-464

[23] K. J. Fujimoto, S. Hayashi Journal of the American Chemical Society 2009, 131(40), 14152-14153

[24] T. Förster, Modern Quantum Chemistry, Vol III, Academic Press, New York, 1965
[25] V. Perlík, J. Seibt, L. J. Cranston, R. J. Cogdell, C. N. Lincoln, J. Savolainen, F. Šanda, T. Mancal, J. Hauer, The Journal of Chemical Physics 2015, 142(21), 212434

[26] K. C. Hasson, F. Gai, P. A. Anfinrud, Proceedings of the National Academy of Sciences 1996, 93(26), 15124-15129.

[27] D. Polli, P. Altoè, O. Weingart, K. M. Spillane, C. Manzoni, D. Brida, G. Tomasello, G. Orlandi, P. Kukura, R. A. Mathies, M. Garavelli, G. Cerullo, Nature 2010, 467, 440

[28] F. V. de A. Camargo, L. Grimmelsmann, H. L. Anderson, S. R. Meech, I. A. Heisler, Physical Review Letters 2017, 118(3)

[29] E. S. Imasheva, S. P. Balashov, J. M. Wang, E. Smolensky, M. Sheves, J. K. Lanyi, Photochemistry and Photobiology 2008, 84(4), 977-984

[30] J. Réhault, M. Maiuri, A. Oriana, G. Cerullo, Review of Scientific Instruments 2014, 85, 123107

[31] D. Brida, C. Manzoni, G. Cerullo, Optical Letters 2012, 37, 3027-3029 
WILEY-VCH

\section{FULL PAPER}

The carotenoid-to-retinal excitation energy transfer in xanthorhodopsin is time-resolved by pump-probe and two-dimensional electronic spectroscopy techniques. An excess of signal amplitude at the energy transfer related cross-peak, with respect to reduced xanthodhodopsin, is a direct evidence of the process.

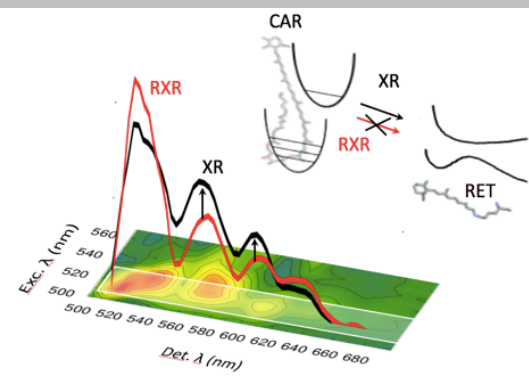

Francesco Segatta, Itay Gdor, Julien Réhault, Simone Taioli, Noga Friedman, Mordechai Sheves, Ivan Rivalta, Sanford Ruhman, * Giulio Cerullo, ${ }^{*}$ and Marco Garavelli*

Page No. - Page No.

Ultrafast Carotenoid to Retinal Energy Transfer in Xanthorhodopsin Revealed by the Combination of Transient Absorption and Two Dimensional Electronic Spectroscopy 